\title{
An Approach to Incremental Design of Distributed Embedded Systems
}

Pop, Paul; Eles, Petru; Pop, Traian; Peng, Zebo

Published in:

A C M / I E E E Design Automation Conference. Proceedings

Publication date:

2001

Document Version

Publisher's PDF, also known as Version of record

Link back to DTU Orbit

Citation (APA):

Pop, P., Eles, P., Pop, T., \& Peng, Z. (2001). An Approach to Incremental Design of Distributed Embedded Systems. A C M / I E E E Design Automation Conference. Proceedings, 450-455.

\section{General rights}

Copyright and moral rights for the publications made accessible in the public portal are retained by the authors and/or other copyright owners and it is a condition of accessing publications that users recognise and abide by the legal requirements associated with these rights.

- Users may download and print one copy of any publication from the public portal for the purpose of private study or research.

- You may not further distribute the material or use it for any profit-making activity or commercial gain

- You may freely distribute the URL identifying the publication in the public portal

If you believe that this document breaches copyright please contact us providing details, and we will remove access to the work immediately and investigate your claim. 


\title{
An Approach to Incremental Design of Distributed Embedded Systems
}

\author{
Paul Pop, Petru Eles, Traian Pop, Zebo Peng \\ Dept. of Computer and Information Science, Linköping University \\ \{paupo, petel, trapo, zebpe\}@ida.liu.se
}

\begin{abstract}
In this paper we present an approach to incremental design of distributed embedded systems for hard real-time applications. We start from an already existing system running a set of applications and the design problem is to implement new functionality so that the already running applications are not disturbed and there is a good chance that, later, new functionality can easily be added to the resulted system. The mapping and scheduling problem are considered in the context of a realistic communication model based on a TDMA protocol.
\end{abstract}

\section{INTRODUCTION}

In this paper we concentrate on aspects related to the synthesis of distributed embedded systems for hard real-time applications. There are several complex design steps to be considered during the development of such a system: the underlying architecture has to be allocated (which implies the allocation of components like processors, memories, and busses together with the decision on a certain interconnection topology), tasks and communication channels have to be mapped on the architecture, and all the activities in the system have to be scheduled. The design process usually implies an iterative execution of these steps until a solution is found such that the resulted system satisfies certain design constraints [7].

Several notable results have been reported, aimed at supporting the designer with methodologies and tools during the hardware/ software cosynthesis of embedded systems. Initially, researchers have considered architectures consisting of a single programmable processor and an ASIC. Their goal was to partition the application between the hardware and software domain, such that performance constraints are satisfied while the total hardware cost is kept at a minimum $[8,6,10,4]$. Currently, similar architectures are becoming increasingly interesting, with the ASIC replaced by a dynamically reconfigurable hardware coprocessor [14].

Distributed embedded systems with multiple processing elements are becoming common in various application areas ranging from multimedia to robotics, industrial control, and automotive electronics. In [19] allocation, mapping, and scheduling are formulated as a mixed integer linear programming (MILP) problem. A disadvantage of this approach is the complexity of solving the MILP model. Therefore, alternative problem formulations and solutions based on efficient heuristics have been proposed [21, 13, 22, 3, 1, 2].

Although much of the above work is dedicated to specific aspects of distributed systems, researchers have often ignored or very much simplified issues concerning the communication infrastructure. One notable exception is [20], in which system synthesis is discussed in the context of a distributed architecture based on arbitrated busses. Many efforts dedicated to communication synthesis have concentrated on the synthesis support for the communication infrastructure but without considering hard real-time constraints and system level scheduling aspects [11, 16, 17].

Another characteristic of research efforts concerning the codesign of embedded systems is that authors concentrate on the design, from scratch, of a new system optimized for a particular application. For many application areas, however, such a situation is extremely uncommon and only rarely appears in design practice. It is much more likely that one has to start from an already existing system running a certain application and the design problem is to implement new functionality on this system. In such a context it is very important to operate no (or as few as possible) modifications to the already running application. The main reason for this is to avoid unnecessarily large design and testing times. Performing modifica- tions on the (potentially large) existing application increases design time and, even more, testing time (instead of only testing the newly implemented functionality, the old application, or at least a part of it, has also to be retested). However, this is not the only aspect to be considered. Such an incremental design process, in which a design is periodically upgraded with new features, is going through several iterations. Therefore, after new functionality has been implemented, the resulting system has to be structured such that additional functionality, later to be mapped, can easily be accommodated.

The contribution of this paper is twofold. First, we consider mapping and scheduling for hard real-time embedded systems in the context of a realistic communication model. Because our focus is on hard real-time safety critical systems, communication is based on a time division multiple access (TDMA) protocol as recommended for applications in areas like, for example, automotive electronics [12]. For the same reason we use a non-preemptive static task scheduling scheme. We accurately take into consideration overheads due to communication and consider, during the mapping and scheduling process, the particular requirements of the communication protocol.

As our main contribution, we have considered, for the first time to our knowledge, the design of distributed embedded systems in the context of an incremental design process as outlined above. This implies that we perform mapping and scheduling of new functionality so that certain design constraints are satisfied and:

a. the already running functionality is not disturbed;

b. there is a good chance that, later, new functionality can easily be mapped on the resulted system.

Supporting such a design process is of critical importance for current and future industrial practice, as the time interval between successive generations of a product is continuously decreasing, while the complexity due to increased sophistication of new functionality is growing rapidly.

The paper is divided into 6 sections. The next section presents the hardware architecture, the process model and a brief introduction of the mapping problem. Section 3 presents the detailed problem formulation and the quality metrics we have introduced. Our mapping strategies are outlined in Section 4, and the experimental results are presented in Section 5. The last section presents our conclusions.

\section{PRELIMINARIES}

\subsection{System Architecture}

We consider architectures consisting of nodes connected by a broadcast communication channel. Every node consists of a CPU, a communication controller, a local memory and an I/O interface to sensors and actuators.

Communication between nodes is based on a TDMA protocol like, for example, the TTP [12] which integrates a set of services necessary for fault-tolerant real-time systems.

The communication channel is a broadcast channel, so a message sent by a node is received by all the other nodes. The bus access scheme is TDMA (Figure 1): each node $N_{i}$ can transmit only during a predetermined time interval, the so called TDMA slot $S_{i}$. In such a slot, a node can send several messages packaged in a frame. A
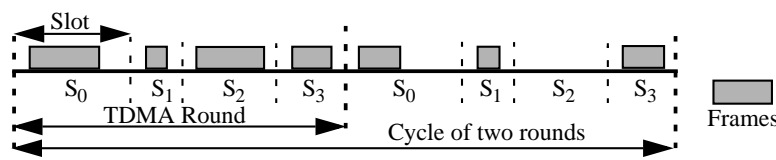

Figure 1. Buss Access Scheme 
sequence of slots corresponding to all the nodes in the architecture is called a TDMA round. A node can have only one slot in a TDMA round. Several TDMA rounds can be combined together in a cycle that is repeated periodically.

Every node has a communication controller that implements the protocol services, and runs independently of the node's CPU. The communication controller provides each CPU with a timer interrupt based on a local clock, synchronized with the local clocks of the other nodes. Thus, a global time-base of known precision is created throughout the system.

We have designed a software architecture which runs on the $\mathrm{CPU}$ in each node, and which has a real-time kernel as its main component. Each kernel has a schedule table that contains all the information needed to take decisions on activation of processes and transmission of messages, based on the current value of time. For more details about the software architecture and the message passing mechanism the reader is referred to [18].

\subsection{The Process Graph}

As an abstract model for system representation we use a directed, acyclic, polar graph $G(V, E)$. Each node $P_{i} \in V$ represents one process. An edge $e_{i j} \in E$ from $P_{i}$ to $P_{j}$ indicates that the output of $P_{i}$ is the input of $P_{j}$. A process can be activated after all its inputs have arrived and it issues its outputs when it terminates. Once activated, a process executes until it completes. Each process graph $G$ is characterized by its period $T_{G}$ and its deadline $D_{G} \leq T_{G}$. The functionality of an application is described as a set of process graphs.

\subsection{Application Mapping}

Considering a system architecture like the one presented in section 2.1 , the mapping of a process graph $G(V, E)$ is given by a function $M: V \rightarrow P E$, where $P E=\left\{N_{l}, N_{2}, . ., N_{\text {npe }}\right\}$ is the set of nodes (processing elements). For a process $P_{i} \in V, M\left(P_{i}\right)$ is the node to which $P_{i}$ is assigned for execution. Each process $P_{i}$ can potentially be mapped on several nodes. Let $\mathcal{N}_{P i} \subseteq P E$ be the set of nodes to which $P_{i}$ can potentially be mapped. For each $N_{i} \in \mathcal{N}_{P i}$, we know the worst case execution time $t_{P_{i}}^{N i}$ of process $P_{i}$, when executed on $N_{i}$.

In order to implement an application, represented as a set of process graphs, the designer has to map the processes to the system nodes and to derive a schedule such that all deadlines are satisfied. We first illustrate some of the problems related to mapping and scheduling, in the context of a system based on a TDMA communication protocol, before going on to explore further aspects specific to an incremental design approach.

Let us consider the example in Figure 2 where we want to map an application consisting of four processes $P_{1}$ to $P_{4}$, with a period and deadline of $50 \mathrm{~ms}$. The architecture is composed of three nodes that communicate according to a TDMA protocol, such that $N_{i}$ transmits in slot $S_{i}$. According to the specification, processes $P_{1}$ and $P_{3}$ are constrained to node $N_{1}$, while $P_{2}$ and $P_{4}$ can be mapped on nodes $N_{2}$ or $N_{3}$, but not $N_{l}$. The worst case execution times of processes on each potential node, the size $m_{i, j}$ of the messages passed between $P_{i}$ and $P_{j}$, and the sequence and size of TDMA slots, are presented in Figure 2.

In [5] we have shown that by considering the communication protocol during scheduling, significant improvements can be made to the schedule quality. The same holds true in the case of mapping. Thus, if we are to map $P_{2}$ and $P_{4}$ on the faster processor $N_{3}$, the resulting schedule length (Figure $2 \mathrm{a}$ ) will be $52 \mathrm{~ms}$ which does not meet the deadline. However, if we map $P_{2}$ and $P_{4}$ on the slower processor $\mathrm{N}_{2}$, the schedule length (Figure $2 \mathrm{~b}$ ) is $48 \mathrm{~ms}$, which is the best possible solution and meets the deadline. Note, that the total traffic on the bus is the same for both mappings and the initial processor load is 0 on both $N_{2}$ and $N_{3}$. This result has its explanation in the impact of the communication protocol. $P_{3}$ cannot start before receiving messages $m_{2,3}$ and $m_{4,3}$. However, slot $S_{2}$ corresponding to node $N_{2}$ precedes in the TDMA round slot $S_{3}$ on which node $N_{3}$ communicates. Thus, the messages which $P_{3}$ needs are available sooner in the case $P_{2}$ and $P_{4}$ are, counter-intuitively, mapped on the slower node.

But finding a valid schedule is not enough if we are to support an incremental design process as discussed in the introduction. In this case, starting from a valid design, we have to improve the mapping and scheduling so that not only the design constraints are satisfied, but also there is a good chance that, later, new functionality can easily be mapped on the resulted system.

To illustrate the role of mapping and scheduling in the context of an incremental design process, let us consider the example in Figure 3. With black we represent the already running set of applications $\psi$ while the current application $\Gamma_{\text {current }}$ to be mapped and scheduled is represented in grey. We consider a single processor, and we present three possible scheduling alternatives for the current application. Now, let us suppose that in future a third application, $\Gamma_{\text {future }}$, has to be mapped on the system. In Figure $3, \Gamma_{\text {future }}$ is depicted in more detail, showing the two processes $P_{1}$ and $P_{2}$ it is composed of. We can observe that the new application can be scheduled only in the first two cases, presented in Figure $3 a$ and $b$. If $\Gamma_{\text {current }}$ has been implemented as in Figure $3 \mathrm{c}$, we are not able to schedule process $P_{2}$ of $\Gamma_{\text {future }}$. The way our current application is mapped and scheduled will influence the likelihood of successfully mapping additional functionality on the system without being forced to redesign and test already running applications.

\section{PROBLEM FORMULATION}

We model an application $\Gamma_{\text {current }}$ as a set of process graphs $G_{i} \in \Gamma_{\text {current }}$, each with a period $T_{G i}$ and a deadline $D_{G i} \leq T_{G i}$. For each process $P_{i}$ in a process graph we know the set $\mathcal{N}_{P i}$ of potential nodes on which it could be mapped and its worst case execution time on each of these nodes. The underlying architecture, as presented in section 2.1, is based on a TDMA protocol. We consider a non-preemptive static cyclic scheduling policy for both processes and message passing.

Our goal is to map and schedule an application $\Gamma_{\text {current }}$ on a system that already implements a set $\psi$ of applications so that:

a.the constraints on $\Gamma_{\text {current }}$ are satisfied without any modification on the implementation of the set of applications $\psi$;

b. new applications $\Gamma_{\text {future }}$ can be mapped on the resulting system.

If no solution is possible that satisfies a) (the algorithm IM discussed in section 4 fails) we have to change the scheduling and possibly the mapping of applications in $\psi$ in order to meet the constraints on $\Gamma_{\text {current }}$. However, even with serious modifications performed on $\psi$, it is still possible that certain constraints are not satisfied. In this case the hardware architecture has to be changed. In this paper we will not discuss the modification of the running applications or of the hardware architecture. We will concentrate on the situation where a possible mapping and scheduling which satisfies requirement a) can be found and this solution has to be further improved by considering requirement $b$ ).

In order to achieve our goal we need certain information to be available concerning the set of applications $\psi$ as well as the possi-

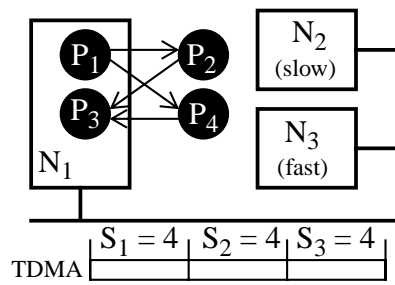

\begin{tabular}{|c|c|c|c|}
\hline & $\mathrm{N}_{1}$ & $\mathrm{~N}_{2}$ & $\mathrm{~N}_{3}$ \\
\hline $\mathrm{P}_{1}$ & $4 \mathrm{~ms}$ & - & - \\
\hline $\mathrm{P}_{2}$ & - & $12 \mathrm{~ms}$ & $8 \mathrm{~ms}$ \\
\hline $\mathrm{P}_{3}$ & $4 \mathrm{~ms}$ & - & - \\
\hline $\mathrm{P}_{4}$ & - & $12 \mathrm{~ms}$ & $8 \mathrm{~ms}$ \\
\hline $\mathrm{m}_{1,2}=\mathrm{m}_{1,4}=\mathrm{m}_{2,3}=\mathrm{m}_{4,3}=4$
\end{tabular}

$\mathrm{N}_{1} \quad \mathrm{P}_{1}$

$\mathrm{N}_{3}$

Bus

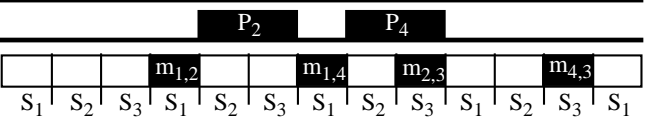

a) Processes $P_{2}$ and $P_{4}$ are mapped on the fast node

$\mathrm{N}_{1}$
$\mathrm{~N}_{2}$

Bus $\mathrm{P}_{1}$

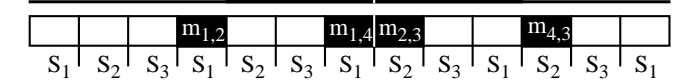

b) Processes $P_{2}$ and $P_{4}$ are mapped on the slow node

Figure 2. Mapping and Scheduling Example 
ble future applications $\Gamma_{\text {future }}$. We assume that the only information available on the existing applications $\psi$ consists of the local schedule tables for each node. This means that we know the activation time for each process on the respective node and its worst case execution time. As for messages, their length as well as their place in the particular TDMA frame are known.

The $\Gamma_{\text {current }}$ application can interact with the previously mapped applications $\psi$ by reading messages generated on the bus by processes in $\psi$. In this case, the reading process has to be synchronized with the arrival of the message on the bus, which is easy to solve during scheduling of $\Gamma_{\text {current }}$.

What do we suppose to know relative to the family $\Gamma_{\text {future }}$ of applications which do not exist yet? Given a certain limited application area (e.g. automotive electronics), it is not unreasonable to assume that, based on the designers' previous experience, the nature of expected future functions to be implemented, profiling of previous applications, available uncomplete designs for future versions of the product, etc., it is possible to characterize the family of applications which possibly could be added to the current implementation. This is an assumption which is basic for the concept of incremental design. Thus, we consider that, relative to the future applications, we know the set $S_{t}=\left\{t_{\text {min }}, \ldots t_{i}, \ldots t_{\max }\right\}$ of possible worst case execution times for processes, and the set $S_{b}=\left\{b_{\text {min }}, \ldots b_{i}, \ldots b_{\text {max }}\right\}$ of possible message sizes. We also assume that over these sets we know the distributions of probability $f_{S t}(t)$ for $t \in S_{t}$ and $f_{S b}(b)$ for $b \in S_{b}$. For example, we might have worst case execution times $S_{t}=\{50,100,200,300,500 \mathrm{~ms}\}$. If there is a higher probability of having processes of $100 \mathrm{~ms}$, and a very low probability of having processes of $300 \mathrm{~ms}$ and $500 \mathrm{~ms}$, then our distribution function $f_{S t}(t)$ could look like this: $f_{S t}(50)=0.20$, $f_{S t}(100)=0.50, f_{S t}(200)=0.20, f_{S t}(300)=0.05$, and $f_{S t}(500)=0.05$.

Another information is related to the period of process graphs which could be part of future applications. In particular, the smallest expected period $T_{\min }$ is assumed to be given, together with the expected necessary processor time $t_{\text {need }}$, and bus bandwidth $b_{\text {need }}$, inside such a period $T_{\min }$. As will be shown later, this information is treated in a flexible way during the design process and is used in order to provide a fair distribution of slacks.

The execution times in $S_{t}$ as well as $t_{\text {need }}$ are considered relative the slowest node in the system. All the other nodes are characterized by a speedup factor relative to this slowest node. A normalization with these factors is performed when computing the metrics $C_{1}^{P}$ and $C_{2}^{P}$ discussed in the following section.

For the sake of simplifying the discussion, we will not address here the memory constraints during process mapping and the implications of memory space in the incremental design process.

\subsection{Quality Metrics}

A designer will be able to map and schedule an application $\Gamma_{\text {future }}$ on top of a system implementing $\psi$ and $\Gamma_{\text {current }}$ only if there are sufficient resources available. In our case, the resources are processor time and the bandwidth on the bus. In the context of a non-preemptive static scheduling policy, having free resources translates into having free time slots on the processors and having space left for messages in the bus slots. We call these free slots of available time on the processor or on the bus, slack. It is to be noted that the total quantity of computation and communication power available on our system after we have mapped and scheduled $\Gamma_{\text {current }}$ on top of $\psi$ is the same regardless of the mapping and scheduling policies used. What depends on the mapping and scheduling strategy is the distribution of slacks along the time line and the size of the individual slacks. It is exactly this size and distribution of the slacks that characterizes the quality of a certain design alternative. In this section we introduce two criteria in order to reflect the degree to which one design alternative meets the requirement b) presented
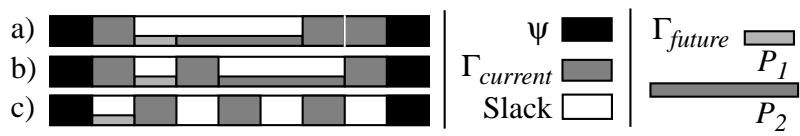

Figure 3. Example for the First Design Criterion

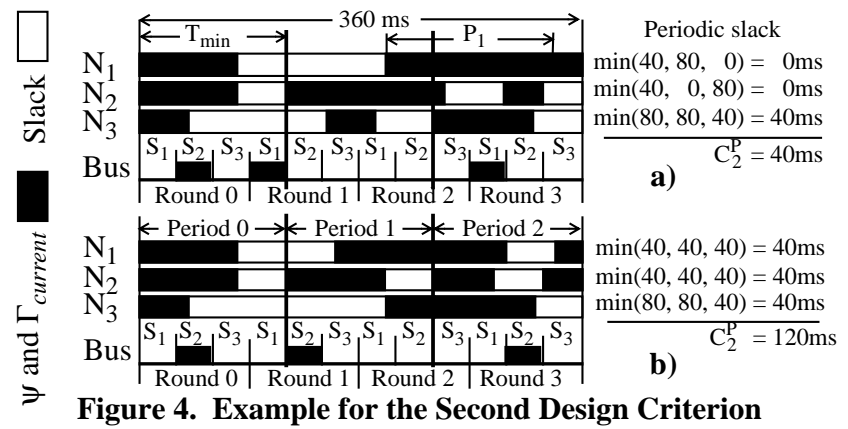

above. For each criterion we provide metrics which quantify the degree to which the criterion is met. The first criterion reflects how well the resulted slack sizes fit to a future application, and the second criterion expresses how well the slack is distributed in time.

\subsubsection{Slack Sizes (the first criterion)}

The slack sizes resulted after implementation of $\Gamma_{\text {current }}$ on top of $\psi$ should be such that they best accommodate a given family of applications $\Gamma_{\text {future }}$, characterized by the sets $S_{t}, S_{b}$ and the probability distributions $f_{S t}$ and $f_{S b}$, as outlined before.

Let us consider the example in Figure 3, where we have a single processor and the applications $\psi$ and $\Gamma_{\text {current }}$ are already mapped. Suppose that application $\Gamma_{\text {future }}$ consists of the two processes $P_{1}$ and $P_{2}$. It can be observed that the best configuration, taking in consideration only slack sizes, is to have a contiguous slack. Such a slack, as depicted in Figure 3a, will best accommodate any future application. However, in reality it is almost impossible to map and schedule the current application such that a contiguous slack is obtained. Not only is it impossible, but it is also undesirable from the point of view of the second design criterion, discussed below. As we can see from Figure 3c, if we schedule $\Gamma_{\text {current }}$ so that it fragments too much the slack, it is impossible to fit $\Gamma_{\text {future }}$ because there is no slack that can accommodate process $P_{2}$. A situation as the one depicted in Figure $3 \mathrm{~b}$ is desirable, where the resulted slack sizes can accommodate the characteristics of the $\Gamma_{\text {future }}$ application.

In order to measure the degree to which the slack sizes in a given design alternative fit the future applications, we provide two metrics, $C_{I}^{P}$ and $C_{1}^{m}$. $C_{I}^{P}$ captures how much of the largest future application which theoretically could be mapped on the system if the slacks would be contiguous, can be mapped on the current design alternative. $C_{l}^{m}$ is similar relative to the slacks in the bus slots. The largest application is determined knowing the total size of the available slack, and the characteristics of the application: $S_{t}$, $f_{t}, S_{b}, f_{b}$. For example, if our total slack size on the processors is of $2800 \mathrm{~ms}$ then, considering the numerical example given in section 3 , the largest application will result as having a total of 20 processes: 4 processes of $50 \mathrm{~ms}, 10$ processes (half, $f_{t}(100)=0.50$ ) of $100 \mathrm{~ms}, 4$ of $200 \mathrm{~ms}$, and one of 300 and $500 \mathrm{~ms}$. After we have determined the largest $\Gamma_{\text {future }}$ we apply a bin-packing algorithm [15] using the best-fit policy in which we consider processes as the objects to be packed, and the slacks as containers. The total execution time of unpacked processes relative to the total execution time of the process set gives the $C_{1}^{P}$ metric. The same applies for the $C_{l}^{m}$ metric. Thus, $C_{1}^{P}=0 \%$ in Figure $3 \mathrm{a}$ and $3 \mathrm{~b}$ (both are perfect from the point of view of slack size), and $75 \%$-- the worst case execution time of $P_{2}$ relative the total slack size -- in Figure 3c.

\subsubsection{Distribution of Slacks (the second criterion)}

In the previous section we provided a metric of how well the sizes of the slacks fit a possible future application. A similar metric is needed to characterize the distribution of slacks over time.

Let $P_{i}$ be a process with period $T_{P i}$ that belongs to a future application, and $M\left(P_{i}\right)$ the node on which $P_{i}$ will be mapped. The worst case execution time of $P_{i}$ is $t_{P_{i}}^{M(P i)}$. In order to schedule $P_{i}$ we need a slack of size $t_{P_{i}}^{M(P i)}$ that is available periodically, within a period $T_{P i}$, on processor $M\left(P_{i}\right)$. If we consider a group of processes with period $T$, which are part of $\Gamma_{\text {future }}$, in order to imple- 
ment them a certain amount of slack is needed which is available periodically, with a period $T$, on the nodes implementing the respective processes.

During implementation of $\Gamma_{\text {current }}$ we aim for a slack distribution such that the future application with the smallest expected period $T_{\text {min }}$ and with the minimum necessary processor time $t_{\text {need }}$, and bandwidth $b_{\text {need }}$, can be accommodated.

Thus, for each node, we compute the minimum periodic slack, inside a $T_{\min }$ period. By summing these minimums, we obtain the slack which is available periodically to $\Gamma_{\text {future }}$. This is the $C_{2}^{P}$ metric. The $C_{2}^{m}$ metric characterizes the minimum periodically available bandwidth on the bus and it is computed in a similar way.

In Figure 4 we consider a situation with $T_{\min }=120 \mathrm{~ms}, t_{\text {need }}=80$ $\mathrm{ms}$, and $b_{\text {need }}=40 \mathrm{~ms}$. The length of the schedule table of our system implementing $\psi$ and $\Gamma_{\text {current }}$ is $360 \mathrm{~ms}$. The system consists of three nodes. Let us consider the situation in Figure 4a. In the first period, Period 0 , there are $40 \mathrm{~ms}$ of slack available on node $N_{l}$, in the second period $80 \mathrm{~ms}$, and in the third period no slack is available on $N_{l}$. Thus, the total slack a future application of period $T_{\min }$ can use on node $N_{l}$ is $\min (40,80,0)=0 \mathrm{~ms}$. Neither node $N_{2}$ can provide slack for this application as in Period 1 there is no slack available. However, on $N_{3}$ there are at least $40 \mathrm{~ms}$ of slack available in each period. Thus, with the configuration in Figure $4 \mathrm{a}$ we have $C_{2}^{P}=40 \mathrm{~ms}$, which is not enough to accommodate $t_{\text {need }}=80$ $\mathrm{ms}$. However, in the situation presented in Figure $4 \mathrm{~b}, C_{2}^{P}=120 \mathrm{~ms}$ $>t_{\text {need }}$, and $C_{2}^{m}=60 \mathrm{~ms}>b_{\text {need }}$.

\subsection{Cost Function and Exact Problem Formulation}

In order to capture how well a certain design alternative meets the requirement b) stated in section 3 , the metrics discussed before are combined in an objective function, as follows:

$$
C=w_{1}^{P}\left(C_{1}^{P}\right)^{2}+w_{1}^{m}\left(C_{1}^{m}\right)^{2}+w_{2}^{P} \max \left(0, t_{\text {need }}-C_{2}^{P}\right)+w_{2}^{m} \max \left(0, b_{\text {need }}-C_{2}^{m}\right)
$$

where the metric values are weighted by the constants $w_{i}$. Our mapping and scheduling strategy will try to minimize this function.

The first two terms measure how well a future application fits to the resulted slack sizes. In order to obtain a balanced solution, that favors a good fitting both on the processors and on the bus, we have used the squares of the metrics.

A design alternative that does not meet the second design criterion is not considered a valid solution. Thus, using the last two terms, we strongly penalize the objective function if either $t_{\text {need }}$ or $b_{\text {need }}$ is not satisfied, by using high values for the $w_{2}$ weights.

At this point, we can give an exact formulation to our problem. Given an existing set of applications $\psi$ which are already mapped and scheduled, and an application $\Gamma_{\text {current }}$ to be mapped on top of $\psi$, we are interested to find a mapping and scheduling of $\Gamma_{\text {current }}$ which satisfies all deadlines and minimizes the objective function $C$, considering a family of future applications characterized by the sets $S_{t}$ and $S_{b}$, the functions $f_{S t}$ and $f_{S b}$ as well as the parameters $T_{\text {min }}, t_{\text {need }}$, and $b_{\text {need }}$.

\section{THE MAPPING STRATEGY}

Our mapping and scheduling strategy has two steps. In the first step we try to obtain a mapping with a valid schedule (which is a schedule that meets the deadlines). Starting from such a solution, a second step iteratively improves on the design in order to minimize the objective function $C$. The minimization of the objective function will hopefully lead to the situation where it is possible to map new applications on the resulting system.

For the algorithm Initial Mapping (IM) that constructs an initial mapping with a valid schedule, we used as a starting point the Heterogeneous Critical Path (HCP) algorithm presented in [9]. $\mathrm{HCP}$ is based on the classical list scheduling algorithm, and uses a ready list $L$ of processes ready to execute, i.e. all their predecessors have been scheduled. In each iteration, a process $P_{i}$ is selected from $L$ according to a priority function based on its critical path length $(\mathrm{CP})$, and assigned to the "best processor" $M\left(P_{i}\right)$. Then, process $P_{i}$ is scheduled on $M\left(P_{i}\right)$. For details on the process and processor selections the reader is referred to [9]. We have modified the HCP algorithm to consider during scheduling a set of previous

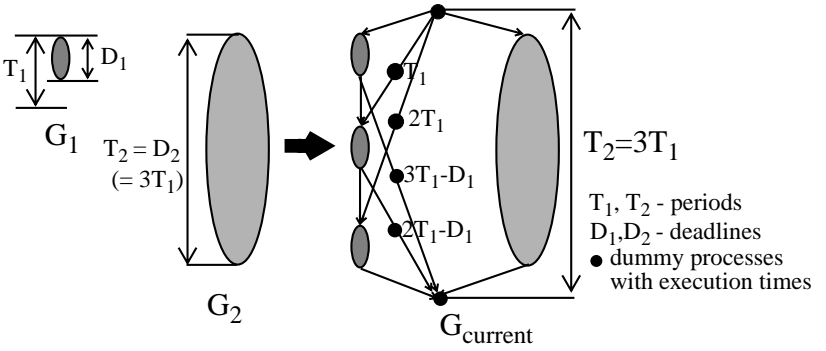

Figure 5. Graph Merging

applications $\psi$ that have already occupied parts of the schedule table, and to schedule the messages according to the TDMA protocol. Furthermore, for the selection of the process from the ready list we have used instead of the CP priority function the MPCP priority function introduced by us in [5]. MPCP takes into consideration the particularities of the communication protocol for calculation of communication delays. These delays are not estimated based only on the message length, but also on the time when slots assigned to the particular node which generates the message, will be available. For the example in Figure 2, our initial mapping algorithm will be able to produce the optimal solution with a schedule length of $48 \mathrm{~ms}$.

However, before using the IM algorithm, two aspects have to be addressed. First, the process graphs $G_{i} \in \Gamma_{\text {current }}$ are merged into a single graph $G_{\text {current }}$, by unrolling of process graphs and insertion of dummy nodes as shown in Figure 5. In addition, we have to consider during scheduling the mismatch between the periods of the already existing system and those of the current application. The schedule table into which we would like to schedule $G_{\text {current }}$ has a length of $T_{\psi}$ which is the global period of the existing system $\psi$. However, the period $T_{\text {current }}$ of $G_{\text {current }}$ can be different from $T_{\psi}$. Thus, before scheduling $G_{\text {current }}$ into the existing schedule table, the schedule table is expanded to the least common multiplier of the two periods. In this context, scheduling $G_{\text {current }}$ means scheduling it into the expanded schedule table inside each period $T_{\text {current }}$. A similar procedure is followed in the case $T_{\text {current }}>T_{\psi}$.

Starting from the valid design produced by IM, our next goal is to improve on the design in order to minimize the objective function $C$. We iteratively improve the design using a transformational approach. A new design is obtained from the current one by performing a transformation called move. We consider the following moves: moving a process to a different slack found on the same node or on a different node, and moving a message to a different slack on the bus. In order to eliminate those moves that will lead to an infeasible design (that violates deadlines), we do as follows. For each process $P_{i}$, we calculate the $A S A P_{i}$ and $A L A P_{i}$ times considering the resources of the given hardware architecture. $A S A P_{i}$ is the earliest time $P_{i}$ can start its execution, while $A L A P_{i}$ is the latest time $P_{i}$ can start its execution without causing the application to miss its deadline. When moving $P_{i}$ we will consider slacks on the target processor only inside the $\left[A S A P_{i}, A L A P_{i}\right]$ interval. The same reasoning holds for messages, with the addition that a message can only be moved to slacks belonging to the same slot number, corresponding to the sender node. Any violation of the data dependency constraints is rectified by moving processes or messages concerned in an appropriate way.

For the goal of improving a design as stated above, we first propose a Simulated Annealing strategy (SA) [4] that aims at finding the near-optimal mapping and schedule that minimizes the objective function $C$. One of the drawbacks of the SA strategy is that in order to find the near-optimal solution it needs very large computation times. Such a strategy, although useful for the final stages of the system synthesis, cannot be used inside a design space exploration cycle.

Thus, we introduce a Mapping Heuristic (MH), outlined in Figure 6, that aims at finding a good quality solution in a reasonable time. MH starts from an initial design produced by IM and iteratively preforms moves in order to improve the design. Unlike 


\section{MappingHeuristic \\ $\operatorname{ASAP}\left(\Gamma_{\text {current }}\right) ; \operatorname{ALAP}\left(\Gamma_{\text {current }}\right)$-- computes ASAP-ALAP intervals InitialMapping $\left(\psi, \Gamma_{\text {current }}\right)$ \\ repeat -- try to satisfy the second design criterion repeat \\ -- find moves with highest potential to maximize $C_{2}^{\mathrm{P}}$ or $C_{2}^{\mathrm{m}}$ move_set=PotentialMoveC $\mathrm{P}_{2}^{\mathrm{P}} \cup$ PotentialMovec $_{2}^{\mathrm{m}}$ -- select and perform move which improves most $C_{2}^{\mathrm{P}}$ or $C_{2}^{\mathrm{m}}$ move $=$ SelectMoveC $_{2}$ (move_set); Perform (move) untij $\left(C_{2} \geq t_{\text {need }}\right.$ and $\left.C_{2}^{m} \geq b_{\text {need }}\right)$ or limit reached \\ if $C_{2}<t_{\text {need }}$ or $C_{2}^{\mathrm{m}}<b_{\text {need }}$ then end if suggest larger $T_{\text {min }}$ \\ until $C_{2}^{P} \geq t_{\text {need }}$ and $C_{2}^{m} \geq b_{\text {need }}$ \\ repeat -- try to improve the metric of the first design criterion -- find moves with highest potential to minimize $C_{l}^{\mathrm{P}}$ or $C_{l}^{\mathrm{m}}$ move set=PotentialMoveC ${ }_{1}^{\mathrm{P}} \cup$ PotentialMoveC $_{1}^{\mathrm{m}}$ \\ -- select move which improves $w_{1}^{P}\left(C_{1}^{P}\right)^{2}+w_{1}^{m}\left(C_{1}^{m}\right)^{2}$ \\ -- and does not invalidate the second design criterion \\ move $=$ SelectMoveC $_{1}$ (move set); Perform (move $)$ \\ until $w_{1}^{P}\left(C_{1}^{P}\right)^{2}+w_{1}^{m}\left(C_{1}^{m}\right)^{2}$ has not changed or limit reached \\ end MappingHeuristic}

Figure 6. Mapping Heuristic to Support Iterative Design

SA that considers all the neighbors of a solution as potential moves, $\mathrm{MH}$ tries to find those neighbors that have the highest potential to improve the design, without evaluating for each of them the objective function. $\mathrm{MH}$ has two main iterative improvement loops. In the first loop it tries to find a solution that satisfies the second design criterion (section 3.1.2). If such a solution cannot be found, then a larger $T_{\min }$ is proposed to the designer. Proposing a larger $T_{\text {min }}$ means that the most demanding future application with the requirements $t_{\text {need }}$ and $b_{\text {need }}$ that we can accommodate, without modifying the existing applications or changing the architecture, cannot have a period smaller than the suggested value. The second loop tries to improve on the metric of the first design criterion (section 3.1.1), without invalidating the second criterion achieved in the first loop. The loop ends when there is no improvement achieved on the first two terms of the objective function, or a limit imposed on the number of iterations has been reached. The intelligence of the Mapping Heuristic lies in how the potential moves are selected. For each iteration a set of potential moves is selected by the PotentialMoveX functions. SelectMoveX then evaluates these moves with regard to the respective metrics and selects the best one to be performed. We now briefly discuss the four PotentialMoveX functions with the corresponding moves:

PotentialMoveC ${ }_{2}^{\mathrm{P}}$ and PotentialMove $\mathrm{C}_{2}^{\mathrm{m}}$

Consider Figure 4a. In Period 2 on node $N_{1}$ there is no available slack. However, if we move process $P_{1}$ with $40 \mathrm{~ms}$ to the left into Period 1, as depicted in Figure 4b, we create a slack in Period 2 and the periodic slack on node $N_{1}$ will be $\min (40,40,40)=40$, instead of 0 . Potential moves will be the shifting of processes inside their $[A S A P, A L A P]$ interval in order to improve the periodic slack. The move can be performed on the same node or to the less loaded nodes. The same is true for moving messages. For the improvement of the periodic bandwidth on the bus, we also consider movement of processes, trying to place the sender and receiver of a message on the same processor and, thus, reducing the bus load.

PotentialMoveC ${ }_{1}^{\mathrm{P}}$ and PotentialMoveC ${ }_{1}^{\mathrm{m}}$

In order to avoid excessive fragmentation of the slack we will consider moving a process to a position that snaps to another existing process. A process is selected for potential move if it has the
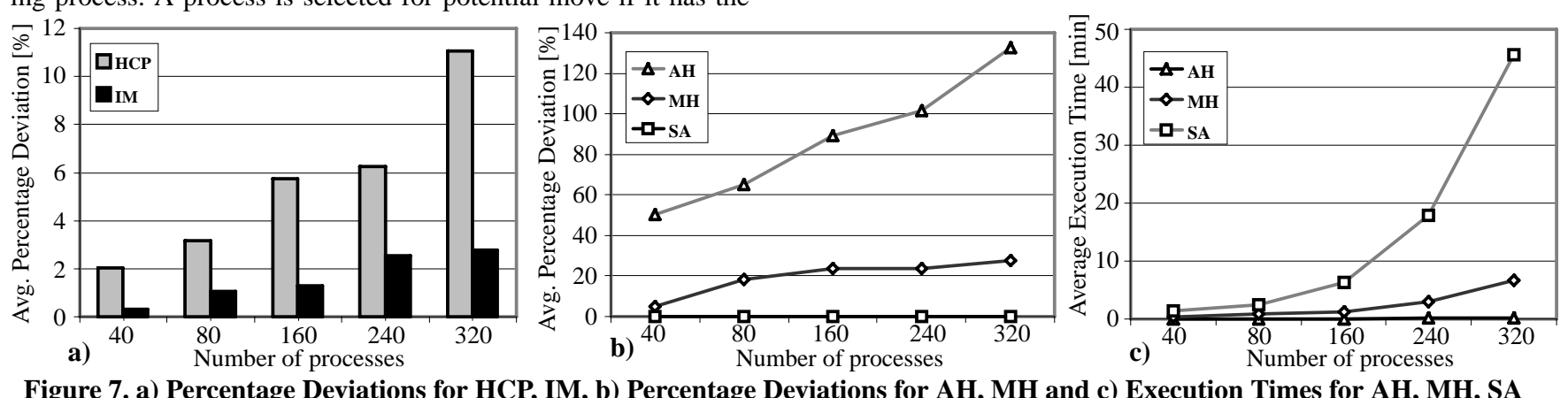

Figure 7. a) Percentage Deviations for HCP, IM, b) Percentage Deviations for AH, MH and c) Execution Times for AH, MH, SA smallest "snapping distance", i.e. in order to attach it to other processes it has to travel the smallest distance inside the schedule table. For a given process such a move is considered both on its node, and to other nodes. We also consider moves that try to increase the individual slacks sizes. Therefore, we first eliminate slack that is unusable: it is too small to hold the smallest process of the future application, or the smallest message. Then, the slacks are sorted in ascending order and the smallest one is considered for improvement. Such improvement of a slack is performed through moving a nearby process or message, but avoiding to create as a result an even smaller individual slack.

\section{EXPERIMENTAL RESULTS}

For evaluation of our mapping strategies we first used process graphs of 40, 160, 240, 320 and 400 processes generated for experimental purpose. 30 graphs were generated for each graph dimension, thus a total of 150 graphs were used for experimental evaluation. We considered an architecture consisting of 10 nodes of different speeds. For the communication channel we considered a transmission speed of $256 \mathrm{kbps}$ and a length below 20 meters. The maximum length of the data field in a bus slot was 8 bytes. All experiments were run on a SUN Ultra 10. Also, throughout the experiments we have considered an existing set of applications $\psi$ consisting of 400 processes, with a schedule table of $6 \mathrm{~s}$ on each processor, and a slack of about $50 \%$ the total schedule size.

The first result concerns the quality of the designs produced by our initial mapping algorithm IM (using the MPCP priority function which considers particularities of the TDMA protocol) compared to the HCP algorithm. We have calculated the average percentage deviations of the schedule length produced with HCP and IM from the length of the best schedule among the two. Results are depicted in Figure 7a. In average, the deviation with IM is 3.28 times smaller than with HCP. The average execution times for both algorithms are under half a second for graphs with 320 processes.

For the next experiments we were interested to investigate the quality of the mapping heuristic MH compared to a so called $a d$ hoc approach $(\mathrm{AH})$ and to the simulated annealing based algorithm SA. The AH approach is a simple, straight-forward solution to produce designs which, to a certain degree, support an incremental process. Starting from the initial valid schedule of length $S$ obtained by IM for the graph $G$ with $N$ processes, AH uses a simple scheme to redistribute the processes inside the $[0, D]$ interval, where $D$ is the deadline of the process graph $G$. AH starts by considering the first process in topological order, let it be $P_{1}$. It introduces after $P_{1}$ a slack of size min(smallest process size of $\Gamma_{\text {future }}$, $(D-S) / N)$, thus shifting all $P_{1}$ 's descendants to the right. The insertion of slacks is repeated for the next process, with the current larger value of $S$, as long as the resulted schedule has an $S \leq D$.

$\mathrm{MH}, \mathrm{SA}$ and $\mathrm{AH}$ have been used to map each of the 150 process graphs on the target system. For each of the resulted designs, the objective function $C$ has been computed. Very long and expensive runs have been performed with the SA algorithm for each graph and the best ever solution produced has been considered as the near-optimum for that graph. We have compared the objective function obtained for the 150 process graphs considering each of the three mapping algorithms. Figure $7 \mathrm{~b}$ presents the average percentage deviation of the objective function obtained with the $\mathrm{MH}$ and $\mathrm{AH}$ from the value of the objective function obtained with the 


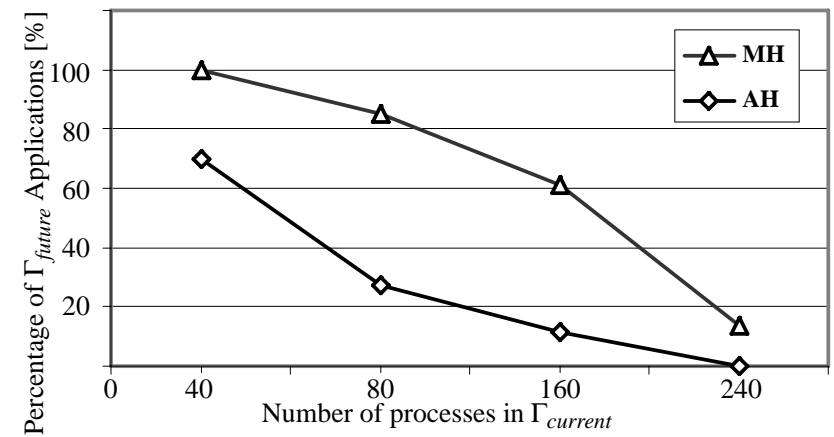

Figure 8. Percentage of $\Gamma_{\text {future }}$ Apps. Successfully Mapped

near-optimal scheme. We have excluded from the results in Figure $7 \mathrm{~b}, 37$ solutions obtained with AH for which the second design criterion has not been met, and thus the objective function has been strongly penalized. The average run-times of the algorithms, in seconds, are presented in Figure 7c. The SA approach performs best in terms of quality at the expense of a large execution time. The execution time can be up to 45 minutes for large graphs of 320 processes. MH performs very well, and is able to obtain good quality solutions in a very short time. AH is very fast, but since it does not address explicitly the two design criteria presented in Section 3 it has the worst quality of solutions, according to the objective function.

The most important aspect of the experiments is determining to which extent the mapping strategies proposed in the paper really facilitate the implementation of future applications. To find this out, we have mapped graphs of 40, 80, 160 and 240 nodes representing the $\Gamma_{\text {current }}$ application on top of $\psi$. After mapping and scheduling each of these graphs we have tried to add a new application $\Gamma_{\text {future }}$ to the resulted system. $\Gamma_{\text {future }}$ consists of a process graph of 80 processes, randomly generated according to the following specifications: $S_{t}=\{20,50,100,150,200 \mathrm{~ms}\}, f_{t}\left(S_{t}\right)=\{10$, $25,45,15,5 \%\}, S_{b}=\{2,4,6,8$ bytes $\}, f_{b}\left(S_{b}\right)=\{20,50,20,10 \%\}$, $T_{\text {min }}=250 \mathrm{~ms}, t_{\text {need }}=100$ and $b_{\text {need }}=20 \mathrm{~ms}$. The experiments have been performed two times, using first $\mathrm{MH}$ and then AH for mapping $\Gamma_{\text {current }}$. In both cases we were interested if it is possible to find a valid implementation for $\Gamma_{\text {future }}$ on top of $\Gamma_{\text {current }}$ using the initial mapping algorithm IM. Figure 8 shows the number of successful implementations in the two cases. In the case $\Gamma_{\text {current }}$ has been mapped with $\mathrm{MH}$, this means using the design criteria and metrics proposed in the paper, we were able to find a valid schedule for $65 \%$ of the total process graphs considered. However, using AH to map $\Gamma_{\text {current }}$ has led to a situation where IM is able to find valid schedules in only $21 \%$ cases. Another observation from Figure 8 is that when the slack size available is large, as in the case $\Gamma_{\text {current }}$ has only 40 processes, it is easy for both $\mathrm{MH}$ and $\mathrm{AH}$ to find a mapping that allows adding future applications. However, as $\Gamma_{\text {current }}$ grows to 160 , only MH is able to find a mapping of $\Gamma_{\text {current }}$ that supports an incremental design process, accommodating more that $60 \%$ of the future applications. If the remaining slack is very small, after we map a $\Gamma_{\text {current }}$ of 240 , it becomes practically impossible to map new applications without modifying the current system.

Finally, we considered an example implementing a vehicle cruise controller (CC) modeled using one process graph. The graph has 32 processes and it was to be mapped on an architecture consisting of 4 nodes, namely: Anti Blocking System, Transmission Control Module, Engine Control Module and Electronic Throttle Module. The period was $300 \mathrm{~ms}$, equal to the deadline. In order to validate our approach, we have considered the following setting. The system $\psi$ consists of 80 processes generated randomly, with a schedule table of $300 \mathrm{~ms}$ and about $40 \%$ slack. The $\mathrm{CC}$ is the $\Gamma_{\text {current }}$ application to be mapped. We have also generated 30 future applications of 40 processes each with the characteristics of the CC, which are typical for automotive applications. By mapping the $\mathrm{CC}$ using $\mathrm{MH}$ we were able to later map 21 of the future applications, while using $\mathrm{AH}$ only 4 of the future applications could be mapped.

\section{CONCLUSIONS}

We have presented an approach to the incremental design of distributed embedded systems for hard real-time applications. Such a design process satisfies two main requirements when adding new functionality: the already running functionality is not disturbed, and there is a good chance that, later, new functionality can easily be mapped on the resulted system. Our approach was considered in the context of a non-preemptive static cyclic scheduling policy and a realistic communication model based on a TDMA scheme.

We have introduced two design criteria with their corresponding metrics, that drive our mapping strategies to solutions supporting an incremental design process. For constructing an initial valid solution, we have shown that it is needed to take into account the features of the communication protocol. Starting from an initial solution, we have proposed two mapping algorithms, SA based on a simulated annealing strategy and $\mathrm{MH}$ an iterative improvement heuristic. SA finds a near-optimal solution at the expense of a large execution time, while $\mathrm{MH}$ is able to quickly produce good quality results.

The approach has been validated through several experiments.

\section{REFERENCES}

[1] J.E. Beck, D.P. Siewiorek, "Automatic Configuration of Embedded Multicomputer Systems", IEEE Trans. on CAD, v17, n.2, 1998, pp. 84-95.

[2] T. Blicke, J. Teich, L. Thiele, "System-Level Synthesis Using Evolutionary Algorithms", Design Automation for Embedded Systems, V4, N1, 1998, pp. 23-58.

[3] B.P. Dave, G. Lakshminarayana, N.K. Jha, "COSYN: Hardware-Software Co-Synthesis of Heterogeneous Distributed Embedded Systems", IEEE Transactions on VLSI Systems, March 1999, pp. $92-104$.

[4] P. Eles, Z. Peng, K. Kuchcinski, A. Doboli, "System Level Hardware/Software Partitioning Based on Simulated Annealing and Tabu Search", Des. Automation for Embedded Syst., V2, N1, 1997, pp. 5-32.

[5] P. Eles, A. Doboli, P. Pop, Z. Peng, "Scheduling with Bus Access Optimization for Distributed Embedded Systems", IEEE Transactions on VLSI Systems, 2000 (to appear).

[6] R. Ernst, J. Henkel, T. Benner, "Hardware-Software Cosynthesis for Microcontrollers", IEEE Design and Test of Computers, vol. 10, Sept. 1993, pp. 64-75.

[7] R. Ernst, "Codesign of Embedded Systems: Status and Trends", IEEE Design\&Test of Comp., April-June, 1998, pp. 45-54.

[8] R. K. Gupta, G. De Micheli, "Hardware-software cosynthesis for digital systems", IEEE Design and Test of Computers, vol. 10, Sept. 1993, pp. 29-41.

[9] P. B. Jorgensen, J. Madsen, "Critical Path Driven Cosynthesis for Heterogeneous Target Architectures," Proc. Int. Workshop on Hardware-Software Co-design, 1997, pp. 15-19.

[10] A. Kalawade, E.A. Lee, "The Extended Partitioning Problem: Hardware/Software Mapping, Scheduling, and Implementation-Bin Selection", Design Automation for Embedded Systems, V2, 1997, pp. 125-163.

[11] P.V. Knudsen, J. Madsen, "Integrating Communication Protocol Selection with Hardware/Software Codesign", IEEE Trans. on CAD, V18, N8, 1999, pp. 1077-1095.

[12] H. Kopetz, G. Grünsteidl, "TTP-A Protocol for Fault-Tolerant Real-Time Systems," IEEE Computer, 27(1), 1994, pp. 14-23.

[13] C. Lee, M. Potkonjak, W. Wolf, "Synthesis of Hard RealTime Application Specific Systems", Design Automation for Embedded Systems, V4, N4, 1999, pp. 215-241.

[14] Yanbing Li, T. Callahan, E. Darnell, R. Harr, U. Kurkure, J. Stockwood, "Hardware-Software Co-Design of Embedded Reconfigurable Architectures"; ACM/IEEE DAC, 2000, 507-512.

[15] S. Martello, P.Toth, "Kanpsack Problems: Algorithms and Computer Implementations", Wiley, 1990.

[16] S. Narayan, D.D. Gajski, "Synthesis of System-Level Bus Interfaces", Proc. Europ. Des. \& Test Conf, 1994, 395-399.

[17] R.B. Ortega, G. Borriello, "Communication Synthesis for Distributed Embedded Systems", Proc. Int. Conf. on CAD, 1998, pp. 437-444.

[18] P. Pop, P. Eles, Z. Peng, "Scheduling with Optimized Communication for Time-Triggered Embedded Systems," Proc. 
Int. Workshop on Hardware-Software Co-design, 1999,78-82.

[19] S. Prakash, A. Parker, "SOS: Synthesis of Application-Specific Heterogeneous Multiprocessor Systems", Journal of Parallel and Distrib. Comp., V16, 1992, pp. 338-351.

[20] D. L. Rhodes, Wayne Wolf, "Co-Synthesis of Heterogeneous Multiprocessor Systems using Arbitrated Communication", Proceeding of the 1999 International Conference on CAD, 1999 , pp. 339 - 342.

[21] W. Wolf, "An Architectural Co-Synthesis Algorithm for Distributed, Embedded Computing systems",IEEE Transactions on VLSI Systems, June 1997, pp. 218 -229.

[22] T. Y. Yen, W. Wolf, "Hardware-Software Co-Synthesis of Distributed Embedded Systems", Kluwer Academic Publ., 1997. 